\title{
Opinion \\ MATHEMATICAL MODELLING IN THE ERA OF CORONAVIRUS (SIX MONTHS IN A NEW REALITY)
}

\author{
VITALY VOLPERT*
}

Received June 30, 2020. Accepted June 30, 2020.

\section{UNPRECEDENTED SITUATION IN SOCIETY AND IN SCIENCE}

The coronavirus epidemic is accompanied by an unprecedented interest in society and in the scientific community for the study of its various aspects and, to a large extent, for mathematical modeling of its development. Classical epidemiological models, known for many years, suddenly became amazingly popular, and their simplicity and clear biological meaning attract a wide audience both in science and beyond. Remarkable solidarity in the scientific community and a desire to contribute to the fight against the epidemic seems to be one of the most striking results of the first half-year of the coronavirus era. New virtual scientific groups and various platforms are being actively created, research programs from national and international organizations are being announced, an active exchange of ideas, information, and results is ongoing. Internet seminars, conferences, meetings are becoming everyday reality and a new, more effective form of work.

At the beginning of the epidemic, it quickly became clear that neither science, nor clinical medicine, nor society as a whole were ready for such a development of events. One has to observe with surprise how countries and entire continents are fascinated by the epidemic progression from afar and wait for their turn, which invariably sets in. And everything occurs according to a well-established scenario: disbelief, lack of protective equipment and medicines, crowded hospitals that can become a source of infection. The general public is mastering a new concept of public immunity and is waiting for a vaccine or a miracle cure from old stocks to appear, and the medieval epidemic-fighting tool - quarantine destroys the economy.

This epidemic has once again shown that we are not able to model and manage complex systems, including collective behavior. Socially important decisions are made, in many cases, by a small group of people based on conjunctural considerations without understanding the long-term consequences. Society, not knowing how to "think" and plan, delegates this right to its representatives, chosen more or less randomly. Unlike Homo sapiens, in which the brain consumes a large part of the energy compared to its weight, the "collective brain" - science and education - plays a much smaller role. Is this why modern society looks more like a dinosaur with large muscles and teeth, a small brain, instinctive behavior and danger of extinction?

\section{SCIENCE IN THE OLD AND NEW REALITY}

The modern organization of science was formed in the 1960s and 1970s, when higher education became publicly available, the number of universities, professors and researchers sharply increased. Education and

Institut Camille Jordan, 43, bd du 11 novembre 1918, 69622 Villeurbanne, France.

* Corresponding author: volpert@math.univ-lyon1.fr 
science had become widespread, natural sciences were in demand by the development of industry and the arms race. Gradually, the enthusiasm and idealism of that era was replaced in science by pragmatism, competition and a business model. Supersonic planes and nuclear reactors were designed, a man visited the moon, but thermonuclear fusion and Mars are still far away. Ecology and sustainable development are important, but vague, and industry and consumption cannot be reduced. The "noble" goals of science became less defined, it began to work more on formal criteria encouraged by the bureaucracy. Grants, articles, citation - three pillars that determine success and career. The H-index becomes the center of the scientific universe, and artificial intelligence, working on the principle of pattern recognition, is trying to replace analytical sciences.

And here comes the coronavirus and shakes this system. It is too early to draw conclusions and predictions. At least now, during the coronacrisis, science is returning to its main purpose - the study of natural phenomena and serving the public interest, but at the same time it is entering a new level of self-organization, which can be seen as another step towards the emergence of collective intelligence and of global self-awareness of the scientific community. It is possible to give an obvious, but conditional analogy with neurons in the brain. A huge number of individual neurons with many connections between them lead to the emergence of consciousness and self-consciousness, functioning independently and not reduced to a simple sum of its elements, as is usually formulated in the definition of complex systems. It is still difficult to predict whether collective intelligence will arise on the basis of human networks, what forms it will take and what functions it will take upon itself, but once it has arisen, it can make a lot of difference in an emerging global world. Ecology, space exploration, resources, medicine, and balanced development of the world community are worthy problems for the world scientific consciousness, which cannot be solved by conventional political, military and financial methods. Not a change in socio-economical formations, but the formation of a rational global world is a question of a new reality. The role of collective intelligence and modelling is to teach society how to calculate its behavior and its consequences.

\section{The EPIDEMIOLOGICAL SITUATION}

The epidemiological situation is developing in different countries in approximately the same scenario. After the appearance of the first cases, 10-20 days elapse, then the exponential growth begins, after another week or two restrictive measures (lockdown, social distancing, isolation of patients, quarantine for possibly infected) begin to be introduced. With the introduction of rather stringent measures, the maximum of confirmed daily cases is achieved after 10-12 days. This time shift corresponds to the incubation period and the onset of serious symptoms when a person goes to the hospital and undergoes testing. If distancing measures are not effective enough, then growth may continue. After reaching the maximum of daily cases, a decrease begins, usually quite slow, although also exponential. When the number of new daily cases and the number of hospitalized ones decreases sufficiently, restrictive measures are weakened and, with time, canceled. Thus, to date, there are three stages in the development of the epidemic: the initial, the introduction of social distance, the weakening and the abolition of distancing (phases 1,2,3). The third phase is accompanied by certain epidemiological measures: masks, distancing in transport and at the places of work and study, etc. In Europe, these measures seem to help contain a new growth in the number of infected people.

Compared with seasonal flu, coronavirus disease has a number of features: a longer incubation period (on average about 5 days, but can reach several weeks), higher mortality (estimated at 2-4\%, although accurate estimates are not yet possible), a large number of asymptotic cases (but possibly transmitting the infection), a large number of cases with a severe course of the disease and complications to other organs (mainly lungs).

These differences in the course of the disease at the individual level determine the differences in the epidemiological situation. In the seasonal flu epidemic, three phases can also be distinguished, but others: slow exponential growth, rapid exponential growth, rapid exponential decrease. The presence of the second phase is clearly visible on epidemiological data, but the explanation for this acceleration should be discussed separately. At the end of the second phase, the number of patients who have developed and developed immunity plus the number of vaccines plus the number of resistant ones due to strong immunity becomes quite large. Simply put, 
there is no one else to get sick and the epidemic is fading. This mechanism of attenuation of the epidemic is associated with the so-called collective immunity.

In a coronavirus epidemic, such a scenario is impossible in modern society, since the healthcare system cannot cope with a large number of cases with a severe course of the disease but it cannot neglect them either. Taking into account that at the first stage of the development of the epidemic, the number of infected increases by 10 times every 8-10 days, one infected will give a million infected in one and a half to two months. Severe forms of the disease are observed in about 1/7 of the total number of registered cases (which grow at the same rate). In a few weeks, any healthcare system with such a development of events will be completely blocked. Moreover, a delay of 10 days with restrictive measures increases 10 times not only the number of infected, but also the number of severe and fatal cases.

Summing up the current epidemiological situation, it is clear that measures of social distancing were necessary, but were taken, as a rule, with a delay. Collective immunity will not be achieved in the near future, since, according to estimates based on serological tests, only $5-10 \%$ of the population possesses antibodies. It is unclear how the seasonality effect will affect and whether the second wave can be avoided. One likely scenario is that the epidemic will intensify in the fall and winter.

\section{EPIDEMiOLOGICAL MODELS}

Classical epidemiological models such as SIR (susceptible-infected-recovered) or SEIR (susceptible-exposedinfected-recovered) are based on the assumption that infection spreads due to the encounters between infected and susceptible individuals. These encounters are supposed to be proportional to the densities of these subpopulations. In the beginning of epidemic, the number of susceptible is close to the total population, and it can be considered as approximately constant. Then we obtain a simple linear ordinary differential equation for the number of infected individuals. Its solution is an exponential function $I(t)=I_{0} e^{\left(R_{0}-1\right) t}$, where $I_{0}$ is the initial number of infected individuals, $t$ is time, $R_{0}$ is the basic reproduction number. If it is larger than 1 , then the number of infected individuals exponentially increases, if it is less than $1, I(t)$ decreases. The measures of social distancing decrease $R_{0}$, and the exponential growth mode changes to the exponential decay mode.

More sophisticated and complete models are often considered with different sub-classes and age groups and taking into account some features of their interaction (contact matrices). They are described by systems of ordinary differential equation. The models are more complex but the main ideas and results remain similar.

The models based on ordinary differential equations do not take into account a possible spatial distribution or heterogeneity of infected individuals and of other population sub-classes. We know, for example, that disease progression can be different in different regions and countries, and epidemic is usually initiated by people coming from other places. Spatial epidemic progression can be described by partial differential equation and by individual based models. Technically, they are more complex, and displacement patterns of infected individuals are difficult to formalize in a mathematical model.

In general, methods of mathematical modelling provide a powerful and well-developed instrument to study epidemics. An important question is whether it is applicable for the coronavirus epidemic from the point of view of its previous development and for the prediction of its further progression. As it is indicated above, epidemiological models well describe exponential growth before lockdown and exponential decay during lockdown. However, the exact growth or decay rates, in the other words, the value of the basic reproduction number $R_{0}$ cannot be predicted in advance. We can only estimate it by fitting the models to the available data. Numerous groups working now in France and in other countries try to predict the epidemic progression after lockdown. As before, the mechanisms working here are well known and understood. The question is about the estimates of parameters. As example, there are studies showing to which extent protective masks reduce the risk of contamination. However, we cannot predict the proportion of the population wearing masks if they are not strictly imposed. The same concerns social distancing including distant work, and public authorities with their way of epidemic management. The latter can strongly influence the epidemic progression and, strangely enough, it represents an important uncertainty factor. There are different modus operandi practiced in different countries, like China, South Korea, Germany, France, Sweden, Brazil, with different results. In the UK, it was changed during 
the first stage of epidemic where "no lockdown" mode was replaced by "lockdown" mode when the authorities had realized an approaching failure of the public health system. It is a rare example where modelling was able to change government's decisions. In big countries, like the US, spatial effects and communication between different states or regions, can be more important than in small and more homogeneous countries. Detailed analysis of epidemic management in different countries will provide valuable information for the future. The cost of this experience is high, both in human lives and damage to economy.

Thus, epidemiological models have their limits in prediction of the future epidemic progression determined by parameters estimation, people behavior, and public authorities, and by the unknown properties of coronavirus, including its seasonality and persistence of acquired immunity.

\section{OTHER QUESTIONS AND MODELS}

The vast majority of works devoted to Covid-19 epidemic deal with epidemiology. At the moment, some other questions, including immune response to coronavirus or pathophysiology of the coronavirus disease basically remain outside of these modelling efforts because of the complexity of these phenomena. We will outline here some directions of possible modelling in these and other directions.

\subsection{Lung alveoli and bronchi}

One of the main complications of the coronavirus disease concerns lungs with different ways of the disease progression in alveoli and in bronchi. Inflammation of the tissue surrounding alveoli during pneumonitis leads to spontaneous blood coagulation in the arterioles. As a result, multiple small clots obstruct blood circulation resulting in the lack of oxygen in the body and possibly in the development of sepsis.

Modelling of blood coagulation including clot formation in flow taking into account biochemical reactions in plasma and platelet aggregation is an active area of research. Numerous simulations and analytical approximations give correct qualitative description of clot growth and the conditions of vessel occlusion. The influence of inflammation on blood coagulation is also investigated, as well as the action of anticoagulant drugs which are now largely used in treatment of coronavirus disease.

In spite of the large body of advanced modelling research on blood coagulation, the possibility of its application in clinical medicine remains limited. The main difficulty here is related to the large variability of patient-dependent parameters of the model. Numerous reaction rate constants of the coagulation reaction in plasma cannot be easily determined for individual patients because of time and cost constraints. As a result, some "averaged" treatment is used in practice, and the ideas of personalized medicine cannot be yet realized. There are some possible ways to fill this gap but they require more time and joint efforts of medical researchers and modelers.

Coronavirus disease progression in the bronchi touches goblet and cilia cells in the epithelial layer. These cells provide the production and motion of mucus along the bronchi serving to eliminate various inert and active particles (including viruses and bacteria) from the lungs. Coronavirus penetrates these cells and reproduces there. It prevents normal functioning of these cells or even kills them in the long. Furthermore, they become the target of adaptive immune response (cytotoxic T-lymphocytes) eliminating infected cells.

As a result, there is less mucus produced, and its motion is less efficient. Moreover, mucus becomes more viscous because of the inflammation which complicates its elimination even more. This creates a vicious cycle between infection, inflammation, mucus production and motion resulting in respiratory problems.

Mathematical modelling of mucus motion was carried out, in particular, in relation with mucoviscidosis. More modelling studies of mucus production and motion are required for the coronavirus disease taking into account the interaction with the infection development and inflammation.

\subsection{Viral infection and immune response}

Immune response to viral infection consists of innate and adaptive components. Cells of innate immune response (macrophages, dendritic cells) and interferon produced by infected cells provide the first barrier for 
the infection development and give some time necessary for the adaptive immune response to produce sufficient amount of antigen specific $\mathrm{T}$ and $\mathrm{B}$ lymphocytes. They eliminate infected cells and produce antibodies neutralizing free virions. This is a generic mechanism of immune response taking place for all viral infections. Immune response to the coronavirus infection has some specific features. The most important of them is the so-called cytokine storm, probably related to the appearance of deficient lymphocytes and leading to the damage of lung tissue due to the excessive immune response.

Mathematical modelling of viral infections is often based on ordinary and delay differential equations for the concentration of virus and immune cells. They allow the determination of the conditions of complete curing and of the development of persistent (chronic) infection taking into account various aspects of immune response and of antiviral treatment. More complete models characterize spatial distribution of viral load in the tissue and in the organism, and virus mutations. As before, one of the main restrictions on the application of mathematical modelling of immune response in clinical medicine is the identification of patient-specific parameters of the model.

The experimental and clinical data on the coronavirus infection are not yet sufficient for the development of virus specific mathematical models. There is some similarity with the previous SARS-CoV virus, and these data can be used for the validation of mathematical models. However, the main progress in modelling of Covid-19 can be expected in the coming years if experiments and clinical medicine provide sufficient amount of data.

\subsection{Other organs, vaccine, treatment}

Coronavirus attacks host cells through the ACE2 receptor expressed in epithelial cells (lungs) and endothelial cells (blood vessels). As a result, different organs (heart, kidney, brain) can be concerned by the disease. Its pathophysiology and treatment should take into account the properties of particular organs. Mathematical modelling of the disease progression in different organs is not yet developed.

Immuno-epidemiological models take into account the interaction of the disease progression at the individual level and at the collective level. They suggest a possible explanation of the epidemic acceleration during seasonal influenza. In the beginning of epidemic, mainly people with weak immune response are concerned. However, when the number of infected becomes sufficiently large, individuals with strong immune response also become susceptible because the initial viral load can increase. This mechanism is generic, and it is important to avoid such scenario in the Covid-19 epidemic.

Among other directions of research, let us mention computer modelling of complex molecules. It allows the reconstruction of virus spikes with a possible application to the development of vaccines and antiviral drugs.

\section{First SiX MONTHS BEHIND, WhAT NEXT?}

First several months of the coronavirus epidemic were quite erratic. Most of the countries were not well prepared, people and authorities could not believe that it would be so serious and then could not properly manage the situation jumping from disbelief to panic. After the first shock, the situation seems to be more stable now in Europe but the level of epidemic is still high in the USA, and it continues its progression in the South America, India, and in some other countries and regions.

The good news is that the total world number of new daily deaths remains approximately constant in spite of growth of new daily infected cases. Probably crucial here is mastering of spontaneous clotting and of cytokine storm, together with using some non-specific antiviral drugs, though the latter remains controversial.

Collective immunity, vaccine, and specific antiviral drugs are not yet there, and it is not clear when they can appear. Media articles write about vaccine testing but there is a large variation in the estimates of its availability.

Mathematical modelling of infectious diseases is one of the elements of multidisciplinary research program together with medical, biological, epidemiological studies. It can help to understand the underlying biological mechanisms, to optimize medical treatment, to give some epidemiological recommendations. However, we should not expect that it will give us a magic formula to stop the epidemic. Scientific research is a long and difficult process with a slow progress but without it, there is no progress at all. We should remember about it not only during dramatic epidemic bursts but also between them. 